\title{
PARTIAL CONVERSION OF HYDROCARBONS TO SYNGAS AND HYDROGEN IN VOLUMETRIC RADIATION BURNERS AS A PROSPECTIVE WAY TO ENHANCE THE PERFORMANCE CHARACTERISTICS OF POWER ENGINES
}

\section{V.S. Arutyunov, V. M. Shmelev, O. V. Shapovalova, A. N. Rakhmetov, and L. N. Strekova}

\author{
Semenov Institute of Chemical Physics \\ Russian Academy of Sciences \\ Kosygina 4, Moscow 119991, Russia
}

\begin{abstract}
New type of syngas generator based on the partial conversion of natural gas (methane) or heavier hydrocarbons in volumetric permeable matrix burners in the conditions of locked infrared (IR) radiation is suggested as a high-productive, adaptable, and rather simple way of syngas and hydrogen production for various low-scale applications including enhancing the performance characteristics of power engines.
\end{abstract}

\section{INTRODUCTION}

Hydrogen is likely to play an important role in power generation, especially with severe constraints on greenhouse gas emissions. But there are two problems that restrict its wide use: high cost of its production and absence of necessary infrastructure for hydrogen supply. An effective short-term solution to face with Green-House Gases problem and, at the same time, to introduce $\mathrm{H}_{2}$ in the fuel market without consistent changes in present time engine technology is to add a moderate content of $\mathrm{H}_{2}$ (up to 20-30\%(vol.)) to natural gas fuel [1] alongside with hydrogen and syngas use in catalytic converters of NOx.

When used in an internal combustion engine, addition of a small amount of hydrogen to natural gas (10-30\%(vol.)) leads to many advantages, due to some particular physical and chemical properties of these fuels. The speed of methane flame propagation is slow while that of hydrogen is about eight times higher.

This is an Open Access article distributed under the terms of the Creative Commons Attribution License 2.0, which permits unrestricted use, distribution, and reproduction in any medium, provided the original work is properly cited. 
When the air/fuel ratio is much higher than for the stoichiometric conditions, combustion of methane is not stable. In consequence of addition of hydrogen to natural gas much more intensive combustion was verified in a wide range of operating conditions (air/fuel ratio, compression ratio, etc.). Therefore, the simultaneous use of methane and hydrogen in internal combustion engines allows working with leaner blends and faster fluxes. It also provides such important benefits as a higher efficiency and lower emission, especially of nitrogen oxides, due to the presence of air quantity higher than the stoichiometric one [1].

Hydrogen is also prospective as gas turbine fuel. In [2], the possibility to burn hydrogen in a large-size, heavy-duty gas turbine designed to run on natural gas is considered as a possible short-term measure to reduce greenhouse emission of power industry. The addition of hydrogen to methane resulted in increase in measured laminar burning velocity and widening the ignition limits. Although at the same equivalence ratio, the NO emission which strongly depends on temperature, increases with hydrogen addition, total equivalence ratio at extinction can be extended. Eventually, NO emission decreased with hydrogen addition. Therefore, the hydrogen addition is a viable method to enhance the flame stability and reduce NO emission simultaneously.

Nowadays, steam and steam-oxygen reforming of natural gas are routinely used in industrial practice to produce syngas and hydrogen. The economical efficiency of these processes sharply falls when the scale of production decreases; so, the possibility of their use for low-scale on-site partial conversion of natural or associated petroleum gases to syngas and hydrogen to use them as additives to power engine fuels is doubtful. Besides, they can hardly be used for distributed production and supply networks of hydrogen for future transportation needs. So, for wider introduction of hydrogen in power and transportation, more simple and convenient low scale technologies of its production are necessary.

In combustion practice, there are widely used radiation burners based on flat permeable matrixes that allow one to improve the efficiency of gas combustion due to a substantially decreasing flame front temperature and, consequently, formation of nitrogen oxides. However, conversion of chemical energy to intense IR radiation in such open systems leads to significant loss of energy from the combustion zone and, as a result of it, makes the combustion limits narrower. The latter hampers the hydrocarbon oxidation (at high fuel-to-oxygen ratios) required for the formation of syngas $\left(\mathrm{H}_{2}\right.$ and $\left.\mathrm{CO}\right)$.

In deep volumetric three-dimensional (3D) matrixes, combustion proceeds in their cavities under the conditions of partial (or almost fully) locked IR radiation that leads to substantial decreasing energy losses by radiation and, thereby, widening the combustion limits in the areas of both fuel-lean [3] and fuel-rich [4] mixtures. In this work, the possibility of syngas and hydrogen production based on natural gas (methane) or propane-butane oxidative conversion at high fuel-to-oxygen ratios in a volumetric permeable matrix is demonstrated. 


\section{EXPERIMENTAL PART}

The scheme of experimental test bench is shown in Fig. 1. Network gas or commercial propane-butane mixtures were used as a fuel whereas air was used as an oxidizer. Gas flows were adjusted according to the readings of rotameters and more precisely measured by gas meters. After the mixer, a homogeneous gas-air mixture of a specified composition was fed into the radiant burner with a deep volumetric matrix.

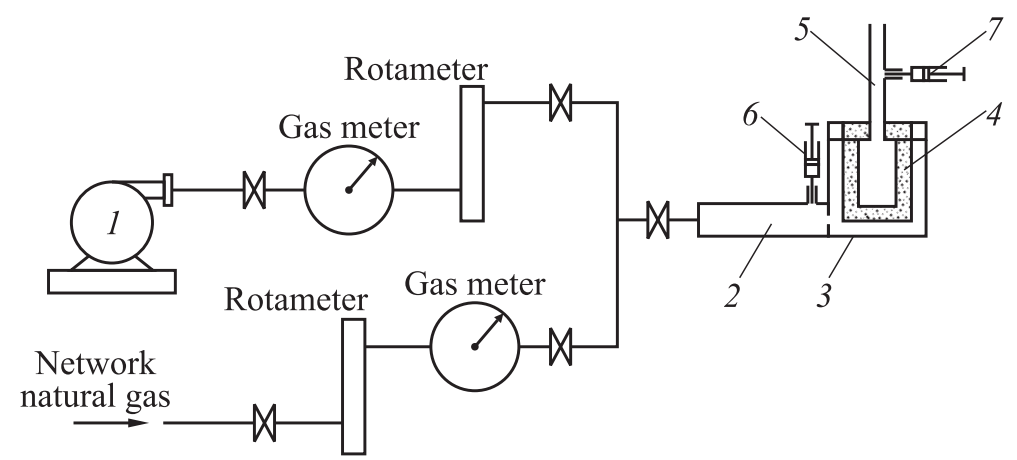

Figure 1 Scheme of experimental test bench: 1 - low-pressure air compressor; $2-$ mixer; 3 - housing of burner; 4 - volumetric matrix; 5 - gas outlet; and 6 and 7 gas sampling points

The principle of performance of syngas generator based on deep radiant volumetric matrix burner is shown in Fig. 2 .

The experiments were performed at atmospheric pressure with a premixed methane-air or propane-butane-air feed. At each experimental set, the fuel-tooxygen ratio was changed by variation of methane flow. In different sets, air flow was varied from 20 to $50 \mathrm{l} / \mathrm{min}$. The content of carbon oxides and oxygen in reaction mixture was permanently monitored by gas analyzer. The composition of mixture $\left(\mathrm{H}_{2}, \mathrm{O}_{2}, \mathrm{~N}_{2}, \mathrm{CO}, \mathrm{CO}_{2}, \mathrm{CH}_{4}\right.$, and some heavier hydrocarbons) was also periodically analyzed by gas chromatograph (GC) equipped with a thermal conductivity (TC) detector and two packed columns (molecular sieves 13X and Porapak Q). Several thermocouples let to measure gas temperature in matrix cavity, temperatures of inner and outer surfaces of matrix, and that of external shell.

The experiments were performed with several different types of $3 \mathrm{D}$ burners fabricated from various materials including perforated ceramic tiles, $\mathrm{Ni}$ or Nichrome foams, and metallic mesh. 


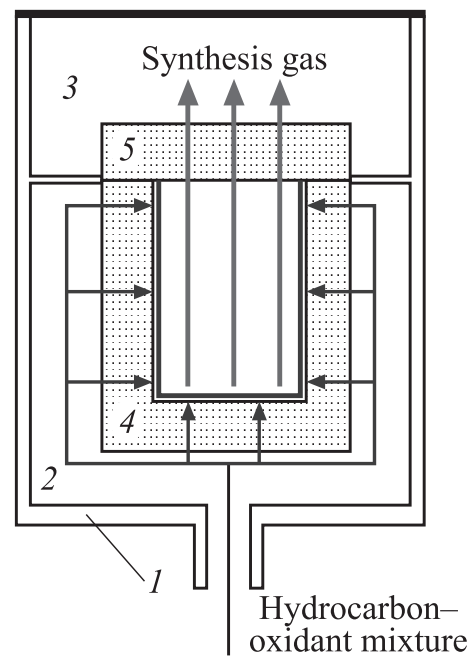

Figure 2 Scheme of syngas generator based on of radiant burner with a deep volumetric matrix: 1 - external shell; 2 - external volume with a fuel-air mixture; 3 gas outlet; 4 - side and bottom permeable walls of the burner; and 5 - permeable cover of the burner

\section{RESULTS AND DISCUSSION}

\subsection{Volumetric Matrix Made of Perforated Ceramic Tiles}

In this series of experiments, a 3D burner with rectangular cavity (internal cross section $80 \times 40 \mathrm{~mm}$, depth $115 \mathrm{~mm}$ ) with matrix walls and bottom fabricated from perforated 15-millimeter-thick ceramic tiles with cylindrical channels of 1.2millimeter diameter was used. The ratio of the total channel cross section to the tile area was 0.25 . It was found that methane as well as propane-butane mixture can be efficiently converted to $\mathrm{H}_{2}$ and $\mathrm{CO}$-containing gas. A stable oxidation was observed in a wide range of $\mathrm{O}_{2} /$ fuel ratios. An example of outlet gas temperature and concentration trends of main reaction products for methane conversion at varied oxygen-to-methane ratio is presented in Fig. 3. Concentrations of $\mathrm{H}_{2}$ and $\mathrm{CO}$ increase at the decreasing oxygen content (oxygen excess coefficient $\left.\alpha=\left[\mathrm{O}_{2}\right]_{0} /\left(2\left[\mathrm{CH}_{4}\right]_{0}\right)\right)$ in the inlet mixture, whereas $\mathrm{CO}_{2}$ content decreases.

It is necessary to note that after the completion of oxygen conversion, concentrations of main products remain nearly constant throughout the height of burner's internal cavity up to its external edge. It can be explained by the fact that at temperatures of $\sim 600-700{ }^{\circ} \mathrm{C}$ during the period of gases reside in the matrix cavity, any gas phase processes with the participation of deep oxidation 


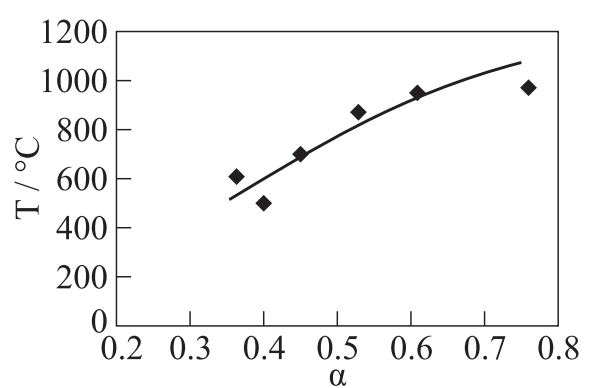

(a)

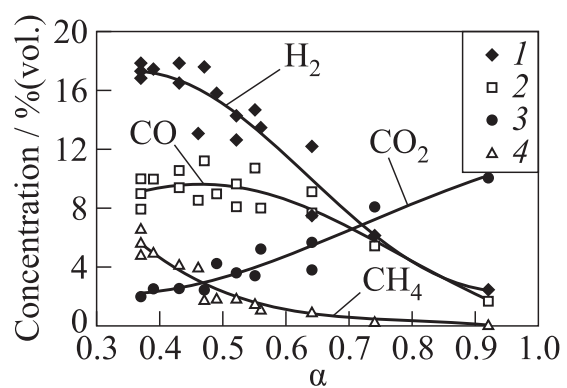

(b)

Figure 3 Experimental outlet gas temperature $(a)$ and concentrations of components in the outlet gas $(b)$ vs. oxygen excess coefficient $\alpha$ (methane-air mixture; air flow $36 \mathrm{l} / \mathrm{min}$; matrix made of perforated ceramic tiles): $1-\mathrm{H}_{2} ; 2-\mathrm{CO} ; 3-\mathrm{CO}_{2}$; and $4-\mathrm{CH}_{4}$

products $\mathrm{CO}_{2}$ and $\mathrm{H}_{2} \mathrm{O}$ advancing the system towards the thermodynamic equilibrium are unreal. Only a monotonic drop in methane concentration which seems to be a result of its thermal pyrolysis under these anaerobic conditions with the formation of heavier condensation products that cannot be registered by the authors' analytical apparatus was observed.

Nevertheless, just near the surface, carbon and hydrogen balances were within $\pm 5 \%$ with oxygen balanced by formation of water which was not determined directly.

The comparison with the results of adiabatic thermodynamic calculations (program GASEQ) demonstrates that for given methane-air ratio, both calcu-



(a)



(b)

Figure 4 Thermodynamic calculations of equilibrium outlet gas temperature $(a)$ and concentrations of principal components in dry gas $(b)$ vs. oxygen excess coefficient $\alpha$ for methane-air mixture 


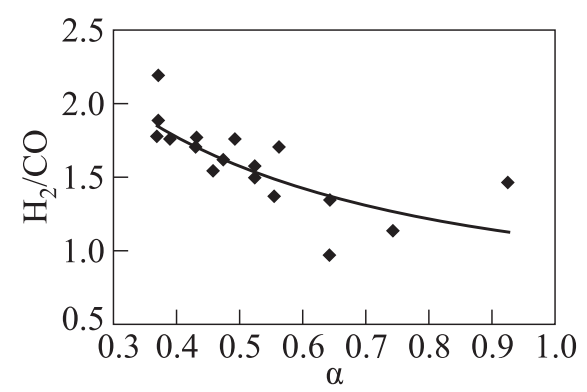

(a)

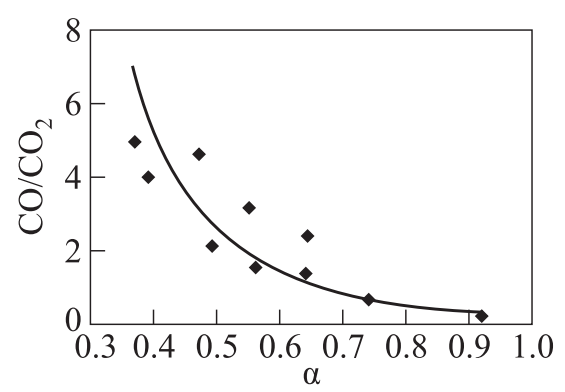

(b)

Figure 5 Dependence of the $\mathrm{H}_{2} / \mathrm{CO}$ ratio $(a)$ and the $\mathrm{CO} / \mathrm{CO}_{2}$ ratio $(b)$ on the oxidizer excess coefficient $\alpha$. Experimental conditions are the same as in Fig. 3

lated equilibrium temperatures and gas compositions (Fig. 4) are close enough to that obtained experimentally. Therefore, in such relatively simple device, even using air as an oxidant, it is possible to obtain high enough concentrations of $\mathrm{H}_{2}$ and $\mathrm{CO}$ close to $20 \%$ and $12 \%$, respectively. But it is worth to note that at $\alpha<0.4$, further increase of $\mathrm{H}_{2}$ and $\mathrm{CO}$ concentrations practically ceased due to the decrease of methane conversion with the fast drop of combustion temperature (see Figs. $3 a$ and $4 a$ ). Such drop of syngas yield is confirmed by the results of kinetic simulation of light hydrocarbons conversion in the flame of diffusion air burner [5]. The possible way to overcome this limitation is the additional preheating of gas mixture at the expense of recuperation of heat of outlet gases or catalytic pretreatment of hydrocarbon fuel before flame front at its interaction with surface of matrix channels. Such possibilities will be considered below.

The dependencies of two most important $\mathrm{H}_{2} / \mathrm{CO}$ and $\mathrm{CO} / \mathrm{CO}_{2}$ ratios characterizing the composition of synthesized products on $\alpha$ are illustrated in Fig. 5 . The $\mathrm{H}_{2} / \mathrm{CO}$ ratio grows monotonically and comes to value $1.8-2.2$ at $\alpha=0.37$ (the equilibrium $\mathrm{H}_{2} / \mathrm{CO}$ ratio is 1.68 at $\alpha=0.4$ and 1.98 at $\alpha=0.3$ ). The $\mathrm{CO} / \mathrm{CO}_{2}$ ratio also increases monotonically and reaches value of 5 at $\alpha=0.37$ (the equilibrium $\mathrm{CO} / \mathrm{CO}_{2}$ ratio is 8.4 at $\alpha=0.4$ ). Hence, in spite of the fact that, on the whole, the thermodynamic equilibrium composition of the products does not attain the main characterizing parameters, the yield of $\mathrm{CO}$ and $\mathrm{H}_{2}$ are close enough to their thermodynamic equilibrium values.

\subsection{Volumetric Matrix Made of Metallic Mesh}

In the next type of converter, the matrix was made of $0.6 \mathrm{~mm}$ Fechral mesh. The inner cylinder cavity of matrix had diameter $62 \mathrm{~mm}$ and height $64 \mathrm{~mm}$ with metallic bottom and cap. The later had an outcome orifice with inserted outcome 
tube with diameter $20 \mathrm{~mm}$ and height $350 \mathrm{~mm}$. The inner working surface of matrix $S$ was about $125 \mathrm{~cm}^{2}$ and that of outcome cross section $S_{1}$ was $30 \mathrm{~cm}^{2}$ with the ratio $S / S_{1}=4.2$. To increase the inner radiation flux, the secondary emitter made of perforated thin-walled metallic cylinder with diameter $52 \mathrm{~mm}$ and height $50 \mathrm{~mm}$ was inserted in the matrix cavity (Fig. 6). Such converter stably operates only at $\alpha>0.48$. Concentrations of $\mathrm{H}_{2}$ and $\mathrm{CO}$ rapidly increase with decreasing of $\alpha$ and at $\alpha \approx 0.5$ are close to adiabatically equilibrium values. For example, at $\alpha=0.5$, experimental concentration of $\mathrm{H}_{2}$ reaches $14 \%$ and that of $\mathrm{CO}-11 \%$ (adiabatically equilibrium values $\sim 20 \%$ and $15 \%$, respectively). The concentration of $\mathrm{CO}_{2}$ falls to $4.2 \%$, while equilibrium value is $\sim 4 \%$.

The maximal selectivity of $\mathrm{H}_{2}$ estimated from hydrogen balance was about 40\%. At methane conversion of $80 \%$, it gives the maximal hydrogen yield $\sim 30 \%$. The maximal selectivity of CO estimated from carbon balance was about $60 \%$, which gives the maximal $\mathrm{CO}$ yield $\sim 50 \%$. These values obtained in relatively simple device, especially in comparison with modern industrial syngas generators, let to hope on good practical prospects of such type of syngas generators.

\subsection{Volumetric Matrix Made of Metallic Foam Tiles}

In this type of converter, the volumetric matrix was assembled from six Chromelnickel foam tiles with porosity of about 0.9 , inserted into common shell (Fig. 7). The inner working surface of matrix was $S=192 \mathrm{~cm}^{2}$ and that of outcome cross section was $S_{1}=41.5 \mathrm{~cm}^{2}$ with ratio $S / S_{1}=4.62$.

Experiments have shown that the converter made of metallic foam operates more stable in comparison with that made of metallic mesh. Even with removed cap, the limiting value of $\alpha$ was about 0.4 . These experiments revealed the importance of the inserted inner perforated thin-walled metallic cylinder that played the role of the secondary emitter, thus significantly increasing the temperature of matrix walls and widening limits of stable combustion of rich mixture. The perforated inner metallic cylinder stabilized combustion at $\alpha>0.4$ and even let 


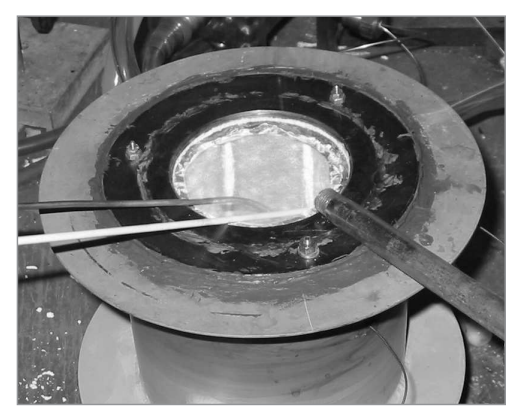

Figure 7 Converter with the matrix made of Chromel-nickel foam tiles operating at power $W=3 \mathrm{~kW}$ during matrix preheating at oxygen excess coefficient $\alpha=1$ (the cap is removed) to obtain some results at $\alpha<0.39$ when combustion in previously preheated at $\alpha$ $=1$ matrix slowly extinguished. It lets to obtain the dependences of burner parameters from air flow and specific stoichiometric thermal load $w$ which is characterized by the density of thermal power on matrix surface at combustion of stoichiometric mixture with the same air flow (Fig. 8). The maximal value of $w$ in the experiments with metallic foam matrix did not exceed $15 \mathrm{~W} / \mathrm{cm}^{2}$ due to limited gas supply while for this type of matrix it can be increased up to $60 \mathrm{~W} / \mathrm{cm}^{2}$.

Figure 8 demonstrates experimental dependence of matrix temperature and concentrations of products from air flow $Q_{a}$ (or specific thermal load $w$ ) at methane conversion in the converter made of metallic foam. The results were obtained after prolonged heating of matrix surface up to $600-700{ }^{\circ} \mathrm{C}$ by combustion of stoichiometric mixture. Then value of $\alpha$ was lowered to 0.39 to conduct the measurements. However, after some time, the temperature of matrix falls below critical value and combustion ceases. It can be seen that at $\alpha \approx 0.39$, $T_{m} \sim 600{ }^{\circ} \mathrm{C}$, and $Q_{a}>30 \mathrm{l} / \mathrm{min}\left(w>10 \mathrm{~W} / \mathrm{cm}^{2}\right)$, concentrations of both $\mathrm{H}_{2}$



(a)

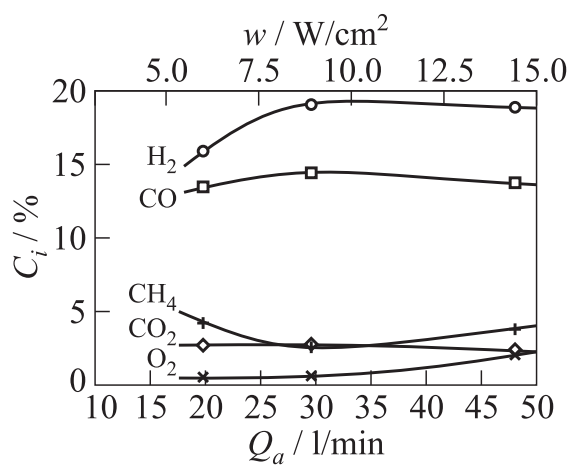

(b)

Figure 8 Experimental dependences of temperature of matrix working (inner) $T_{m}$ and outer $T_{b}$ surfaces $(a)$ and concentrations of products $(b)$ vs. air flow $Q_{a}$ (or specific stoichiometric thermal load $w$ ) at methane conversion in the burner made of Chromelnickel foam $(\alpha \approx 0.39$, extinction mode) 
and $\mathrm{CO}$ are close to equilibrium values. For example, experimentally obtained concentration of $\mathrm{H}_{2}$ reached $20 \%$ and that of $\mathrm{CO} 13 \%$ while equilibrium values are $\sim 25 \%$ and $14 \%$, respectively.

\subsection{Volumetric Matrix with the Recuperation of Heat}

To provide more stable operation at low values of $\alpha$, the recuperation of heat from outlet gases to inlet gas mixture was organized. The air-gas mixture was introduced in the burner through crimped metallic tube situated coaxially along the burner axis. For matrix made of metallic mesh, the heating of inlet gas by hot outlet gas and thus increasing of the initial temperature of mixture let to decrease the stable conversion limit from $\alpha=0.48$ to 0.39. Some additional measures to optimize burner construction and thermal insulation of the external shell let to decrease the stable oper-



Figure 9 The boundary of stable operation and extinction of flame for the converter with matrix made from Chromel-nickel foam (with the recuperation of heat) ation limit for converter with matrix made of metallic foam down to $\alpha=0.35$ (Fig. 9). But at such low values of $\alpha$, the formation of soot observes. It worth to note that for both matrix made of metallic foam (see Fig. 8) and metallic mesh, the dependences of matrix temperature from thermal load are very similar that indicates approximately the same matrix temperatures at equal thermal load.

It is evident (see Fig. 8) that specific thermal load is the determining factor for the extinction of flame. Besides, the limit of stable operation can be widening to lower values $\alpha$ by increasing of heat recuperation, i. e., by increasing the temperature of inlet gas (Fig. 10).

In the converter made of metallic foam, the recuperation of heat provides stable combustion of mixtures with $\alpha$ down to 0.35 . In this case, concentrations of $\mathrm{H}_{2}$ and $\mathrm{CO}$ attain their highest values at the highest specific thermal load, which in the present experiments did not exceed $15 \mathrm{~W} / \mathrm{cm}^{2}$ (see Fig. 10). So, it can be supposed that the further increasing of specific thermal load up to possible value $60 \mathrm{~W} / \mathrm{cm}^{2}$ will increase not only the capacity of this device but as well, alongside with the increasing of the temperature of matrix, the yield of $\mathrm{H}_{2}$ and $\mathrm{CO}$. 


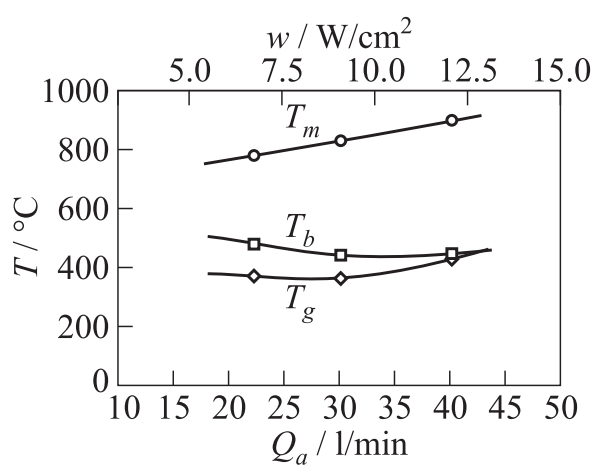

(a)

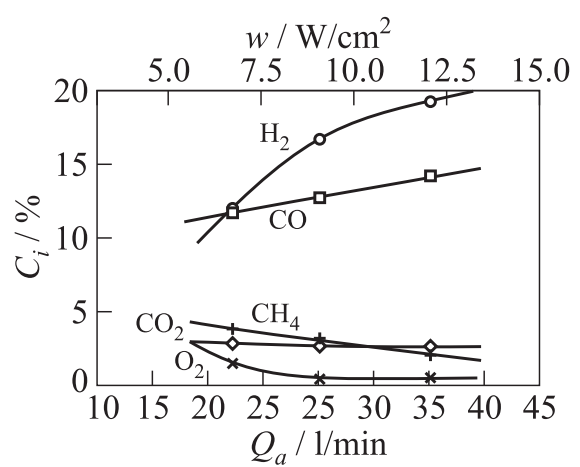

(b)

Figure 10 Experimental dependences of temperature of matrix working (inner) $T_{m}$ and outer $T_{b}$ surfaces $(a)$ and concentrations of products $(b)$ vs. air flow $Q_{a}$ (specific stoichiometric thermal load $w$ ) at methane conversion in the burner made of Chromelnickel foam (recuperation of heat, $\alpha=0.37$, stable mode)

\subsection{Volumetric Matrix with Catalytic Active Surface}

Previously, it was supposed that matrix does not cause any other influence on gas-fuel mixture except its heating. Of course, at temperature above $600{ }^{\circ} \mathrm{C}$ (see Figs. 8 and 10), practically any surface exhibits some catalytic properties. Besides, a treatment of the matrix walls with active catalytic components can lead to a widening of the combustion limit for methane-rich mixtures. At last, as it can be seen from Fig. 3, at low values of $\alpha$, the decreasing of flame front temperature also rapidly decreases the conversion of fuel thus decreasing syngas yield. So, it is possible to expect that the catalytic pretreatment of gas-fuel mixture during its interaction with heated matrix will not only enhance the stability of flame at low $\alpha$ but as well will increase syngas yield.

Investigation of different types of matrix materials was performed in a cylindrical volumetric matrix with inner diameter $50 \mathrm{~mm}$ and height of about $100 \mathrm{~mm}$ made of alundum. The permeable bottom that serves as a matrix was made of 15-millimeter-thick perforated ceramic plate with cylindrical channels of 1.2millimeter diameter.

As it was expected, the treatment of ceramic plate with Pt catalyst led to significant enhancing of rich combustion limit (Fig. 11). But although Pt catalyst widening conversion limits, it simultaneously enhances the yield of products of deep oxidation $\left(\mathrm{CO}_{2}\right.$ and $\left.\mathrm{H}_{2} \mathrm{O}\right)$ thus decreasing the yield of goal products $-\mathrm{H}_{2}$ and $\mathrm{CO}$. So, it is necessary to find milder catalysts capable to convert methane and other hydrocarbon fuels at typical matrix temperatures and contact times 
into some intermediates, e.g., oxygenates, that can be more easily transformed into syngas.

Besides perforated ceramic, several other types of matrix materials, e.g., metallic foams made of Nichrome and $\mathrm{Ni}$ with different porosity (from 60 to $80 \mathrm{ppi}$ ) were tried. But with the exception of $\mathrm{Pt}$ catalyst, the matrix material and the noted difference in porosity had insignificant effect on burner operation and the yield of products.

Taking into account the positive influence of hydrogen addition to fuel on performance of different

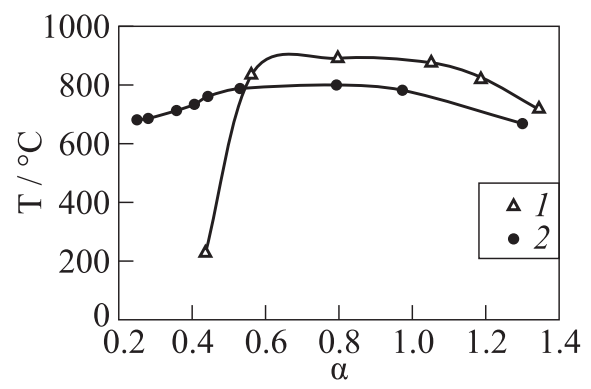

Figure 11 Dependence of outgoing gas temperature vs. oxygen excess coefficient $\alpha$ for methane-air mixture for perforated ceramic matrix (1) and that treated with $\mathrm{Pt}$ catalyst (2) (total gas flow $-6.2 \mathrm{l} / \mathrm{min}$ ) types of power engines, the yield of hydrogen can be enhanced at the expense of CO by its conversion to hydrogen in a water-gas-shift (WGS) reaction [6]. Some experimental sets were also performed with hydrocarbons heavier than methane-propane-butane mixture and evaporated straight-run gasoil. They showed prospective results and well possibility to convert heavier and even liquid hydrocarbons into syngas in such type of converter. This work is under investigation now as well as the investigation of conversion of biogas and some other gases with low heating value.

\section{CONCLUDING REMARKS}

Thus, the combustion of natural gas (methane) and heavier hydrocarbons in volumetric permeable matrix burners in the conditions of locked IR radiation can be considered as a high-productive, adaptable, and rather simple way of syngas or hydrogen (with the use of WGS) production for various low-scale applications including enhancing the performance characteristics of power engines. Due to continuous operation, it is more convenient than filtration combustion in porous media. It is important that the absence of interaction of products with the surface of matrix and very short time of fuel conversion in the flame front let to avoid such serious problem as soot formation. The use of enriched air or oxygen as oxidant and enhanced pressures can further widen the limits of stable operation and fuel conversion thus making such type of converter convenient for many other applications.

There is another global problem to which the use of volumetric permeable matrix burners can be addressed. Due to existing estimates, about 150 billion 
cubic meters of associated oil gas is flared annually worldwide [7]. The main reason is that it is very difficult to use raw associated gas as a fuel in modern types of power engines. Due to high content of heavier hydrocarbons, such gases are very incline to detonation as well as soot and tar formation. Modern technologies of gas treatment are too complex and costly to be applied for usually low fluxes of associated oil gas which makes their flaring economically preferable. But simple enough volumetric permeable matrix burners can effectively convert such low gas fluxes into syngas or hydrogen thus making them more attractive for low-scale and ecologically clean local power supply including use of fuel sells.

\section{REFERENCES}

1. BONG-HY. 2007. Parallel application of blends of natural gas and hydrogen in internal combustion engines and fuel cells. Final Report.

2. Chiesa, P., G. Lozza, and L. Mazzocchi. 2005. Using hydrogen as gas turbine fuel. J. Engng. Gas Turbines Power 127:73-80.

3. Shmelev, V. M., and A. D. Margolin. 2000. On gas mixture burning above the surface of perforated matrix. Russ. J. Chem. Phys. 19(5):36-42.

4. Arutyunov, V.S., V. M. Shmelev, I. N. Lobanov, and G. G. Politenkova. 2010. A generator of synthesis gas and hydrogen based on a radiation burner. Theor. Found. Chem. Engng. 44(1):20-29.

5. Basevich, V. Ya., V.I. Vedeneev, and V.S. Arutyunov. 1996. Study of partial oxidation of hydrocarbons with reference production of synthetic gas. Theor. Found. Chem. Eng. 30(5):456-60.

6. Ratnasamy, C., and J. P. Wagner. 2009. Water gas shift catalysis. Catalysis Reviews 51:325-440.

7. Elvidge, C. D., K. E. Baugh, D. W. Pack, and C. Milesi. 2007. Satellite data estimate worldwide flared gas volumes. Oil Gas J. 12:50-58. 